\title{
Evaluation of New Insecticide Molecules for the Management of Early Shoot Borer (Chilo infuscatellus (Snellen) on Sugarcane
}

\author{
M. Shobharani*, V. Rachappa, Sidramappa and N.M. Sunilkumar \\ Agricultural Research Station, Bidar University of Agricultural Sciences, \\ Raichur, Karnataka, India \\ *Corresponding author
}

\section{A B S T R A C T}

\section{Key words \\ Early shoot borer, \\ Chlorantraniliprole 18.5 \\ SC, Chlorantraniliprole \\ $0.4 \mathrm{GR}$, Management \\ Article Info \\ Accepted: \\ 16 August 2018 \\ Available Online: \\ 10 September 2018}

An experiment was conducted for two successive years during 2013-14 and 2014-15 at Agricultural Research Station, Bidar and Kalburgi, to assess the chemical control of sugarcane early shoot borer (Chilo infuscatellus (Snellen). Nine insecticides namely, Chlorantraniliprole 0.4 GR, Fipronil 0.3 GR, Cartaphydrochloride 4G, Chlorantraniliprole 18.5 SC, Fipronil 5 SC, Flubendiamide 480 SC, Emamectin benzoate 0.5 SG, Chlorpyriphos $20 \mathrm{EC}$ and compared with untreated control using randomized block design with three replications. Significant differences were noticed among the treatments. Among the different insecticides evaluated, Chloranthriniliprole 18.5 SC and Chloranthriniliprole 0.4 GR, were proved superior by recording lowest per cent incidence of early shoot borer and highest cane yield.

\section{Introduction}

Sugarcane (Saccharum officinarum L.) is one of the most important cash and industrial crop in India. Among sugarcane growing countries in the world, India ranks first in the world in the area under sugarcane cultivation and ranks fifth in the world in terms of sugar production. In India, sugarcane occupies an area of $5.01 \mathrm{~m}$ ha and produces $338.96 \mathrm{~m}$ tonnes of cane with an average productivity of 66.99 tones ha. About 35 million farmers in the country depend on the sugarcane for their live hood. Sugarcane yield is markedly influenced by many factors like soil fertility, climate, variety, and cultural practices, prevalence of pests and diseases and environmental stress. Regardless of pronounced development in sugarcane research and expansion in sugar industry, our national average cane yield is 70.3 tons per hectare, whereas the potential between 100120t/ha.

One of the major constraints in maintaining sustainability is losses due to insect pests, as sugarcane is known to be attacked by as many as 212 insect pests and 76 non insect pests in India right from germination to harvest. Among them, borers are the major destructive pests which cause 8 to 10 percent cane yield losses at farmer's level and 10 to 15 percent sugar recovery losses in sugar industries. Among the pest, Gupta (1993) reported more 
than 45 per cent of yield losses in sugarcane are due to infestation by borer pests alone. Among them, the early stem borer Chilo infuscatellus is the most notorious and destructive one. The early shoot borer damages cane crop mainly at formative phase. The young larvae bore down the spindles as also upwards destroying the apical meristem. Consequently the cut of spindle dries up and develops into a conspicuous "dead heart" that can be pulled out easily and emit a rancid odour. The killing of mother shoots and tillers are resulted in to gap. The shoot Borer, Chilo infuscatellus (Snellan) cause economic losses (Avasthy and Tiwari, 1986) from 22-23 per cent in yield, 12 per cent in sugar recovery and 27 per cent in Jaggary. The damage by Chilo infuscatellus ranges from $30-75$ per cent in early stages of the crop (May- June) in subtropical India (Krishnamurthy Rao, 1954). The neglected crop therefore, may suffer heavy losses in tonnage due to this borer species. Keeping in view the economics, importance of the pest and the crop, field studies were carried out to find out the insecticides for management of early shoot borer in sugarcane.

\section{Materials and Methods}

A field experiment was conducted for two successive years during 2014 and 2015 rabisummer at two locations, Agricultural Research Station, Bidar and Kalburgi, to assess the chemical control of sugarcane early shoot borer (Chilo infuscatellus (Snellen). The experiment was laid out in Randomized block design with ten treatments replicated thrice. The Sugarcane Var, Co 86032 was sown at $\mathrm{cm} 90 \times 30 \mathrm{~cm}$ spacing with plot size of $7.5 \times 4 \mathrm{mts}$. and all the recommended package of practices were followed to raise the crop, except plants protection measures. First spray and soil application of insecticides was done at initiation of pest infestation in all the experimental plots. The spraying of insecticides was carried out during morning hours by hand operated knapsack sprayer and granular insecticides were applied in the soil.

Observations on incidence of early shoot borer, Chilo infuscatellus or per cent dead heart was recorded at one day before spray or soil application and 7, 15 and 30 days after each spray or soil application on randomly selected 10 plants /plot. The data was transformed to arcsine values and subjected for Statistical analysis. The cane yield was recorded plot wise at the time of harvest and converted to hectare basis and subjected for statistical analysis.

\section{Results and Discussion}

The results of the experiment carried out at Agricultural Research Station, Bidar for two consecutive years 2013-14 and 2014-15 is pooled and presented in the table 1 . The results of the experiment reveals that, the incidence of early shoot borer ranged from 18.83 to 20.74 per cent early shoot borer damage. Further it was observed that, there was no significant difference with respect to the per cent early shoot borer damage at one day before spraying (DBS), indicating the uniform infestation throughout experimental field.

Among all the treatments, at 15 Days After Spraying (DAS) Chlorantraniliprole 18.5 SC @ $150 \mathrm{ml} / \mathrm{ac}$ and Chlorantraniliprole $0.4 \mathrm{GR}$ @ $8 \mathrm{~kg} / \mathrm{ac}$ found to be significantly superior compared to all other treatments and recorded 4.02 and 4.30 per cent dead hearts respectively. These two treatments were followed by Fipronil 0.3 GR @ 8kg/ac and Cartaphydrochloride 4G @ 5kg/ac which are next in the order of efficacy with 6.22 and 6.47 per cent dead hearts respectively with no significant difference among them. The next best treatments in order of their efficacy were Chlorantraniliprole 18.5 SC @ 60 ml/ac, 
Fipronil 5 SC@ @ml/lt, Flubendiamide 480 SC @ $0.075 \mathrm{ml} / \mathrm{lt}$, Emamectin benzoate 0.5 SG@0.2g/lt, and Chlorpyriphos 20 EC @ 2 $\mathrm{ml} / \mathrm{lt}$ with $9.54,9.65,13.13,13.20$ and 14.92 per cent dead hearts respectively. The dead hearts percentage was significantly low in all the insecticidal treatments as compare to untreated control which recorded 23.98 per cent dead hearts, when observations were recorded at 15 DAS.

The data on early shoot borer incidence recorded at 30 DAS showed the same trend in their efficacy in managing the incidence of early shoot borer.

The early shoot borer incidence recorded at forty five days after treatment showed the lowest early shoot borer incidence in the plots treated with Chlorantraniliprole 18.5 SC @ $150 \mathrm{ml} / \mathrm{ac}$ and Chlorantraniliprole 0.4 GR @ $8 \mathrm{~kg} / \mathrm{ac}$ and were found consistently to be the most promising treatment by recording significantly lowest 1.26 and 1.39 per cent dead hearts respectively. The treatment with Cartaphydrochloride 4G @5kg/ac and Fipronil 0.3 GR @ 8kg/ac are the next in the order of efficacy with 3.99 and 4.11 per cent dead hearts respectively with no significant difference among them. The next best treatments in the order of their efficacy were Chlorantraniliprole 18.5 SC @ 60 ml/ac, Fipronil 5 SC @2ml/lt, Emamectin benzoate 0.5 SG@0.2g/lt, Flubendiamide480 SC @ $0.075 \mathrm{ml} / \mathrm{lt}$ and Chlorpyriphos 20 EC @ 2 $\mathrm{ml} / \mathrm{lt}$ with 7.04, 7.4011.87, 12.16 and 15.41 percent dead hearts respectively. However, untreated control plot recorded the highest per cent dead heart of 29.85 per cent.

\section{Yield}

The significantly highest cane yield was recorded in Chlorantraniliprole 0.4 GR @ 8 kg/ac and Chlorantraniliprole 18.5 SC @150 $\mathrm{ml} / \mathrm{ac}$ with 92.47 tonns/ha and 92.10 tonns/ha respectively. The next best treatments were Fipronil 0.3 GR @ 8kg/ac and Cartaphydrochloride 4G @ 5kg/ac which recorded 84.24 and 83.05 tonns/ha cane yield respectively. These two treatments were followed by Chloranthriniliprole 18.5 SC @ 60 ml/ac, Fipronil 5 SC @ 2ml/lt, Emamectin benzoate0.5 SG@0.2g/lt, Flubendiamide 480 SC @ 0.075 ml/lt and Chlorpyriphos 20 EC @ $2 \mathrm{ml} / \mathrm{lt}$ with the cane yield of 74.84 , $74.54,69.87,67.47$ and 67.07 tonns/ha respectively (Table 1). However, untreated control plot recorded the lowest yield of 61.88 tonns/ha.

\section{B: $\mathbf{C}$ ratio}

Chloranthriniliprole 0.4 GR @ 8 kg/ac and Chloranthriniliprole 18.5 SC @ 150 ml/ac recorded highest $\mathrm{B}: \mathrm{C}$ ratio of 3.70 and 3.63 respectively (Table 2). The next best treatments were and Cartaphydrochloride 4G @ 5kg/ac and Fipronil 0.3 GR @ 8kg/ac which recorded $\mathrm{B}$ : $\mathrm{C}$ ratio of 3.48 and 3.47 respectively. These two treatments were followed by Fipronil 5 SC @ 2ml/lt, Chloranthriniliprole 18.5 SC @ $60 \mathrm{ml} / \mathrm{ac}$, Emamectin benzoate $0.5 \mathrm{SG} @ 0.2 \mathrm{~g} / \mathrm{lt}$, Chlorpyriphos 20 EC @ 2 ml/lt and Flubendiamide 480 SC @ $0.075 \mathrm{ml} / \mathrm{lt}$ with $3.02,2.97,2.73,2.65$ and $2.61 \mathrm{~B}: \mathrm{C}$ ratio respectively. However, untreated control plot recorded the lowest $\mathrm{B}$ : $\mathrm{C}$ ratio of 2.45 .

\section{ARS, Kalburgi}

The results of the experiments conducted at farmers field near ARS, Kalburgi is presented in the table 2. The incidence of early shoot borer during 2013-14 and 2014-15 ranged from 21.75 to 24.15 per cent. Further it was observed that, there was no significant difference with respect to the per cent early shoot borer damage at one day before spraying (DBS), indicating the uniform infestation throughout experimental field. 
Table.1 Evaluation of new molecules against Early Shoot borer on Sugarcane of 2013-14 and 2014-15 at ARS, Bidar (Pooled)

\begin{tabular}{|c|c|c|c|c|c|c|}
\hline \multirow{2}{*}{$\begin{array}{l}\text { Sl. } \\
\text { No. }\end{array}$} & \multirow[t]{2}{*}{ Treatments } & \multicolumn{4}{|c|}{ Early shoot borer incidence ( $\%$ deadheat) } & \multirow{2}{*}{$\begin{array}{l}\text { Yield } \\
\text { (t/ha) }\end{array}$} \\
\hline & & 1DBS & 15 DAS & 30 DAS & 45 DAS & \\
\hline 1 & Chlorantraniliprole 0.4 GR ( $8 \mathrm{Kg} / \mathrm{ac})$ & $\begin{array}{c}19.06 \\
(25.84)\end{array}$ & $\begin{array}{c}4.30 \\
(11.90)\end{array}$ & $\begin{array}{c}1.10 \\
(5.96)\end{array}$ & $\begin{array}{c}1.39 \\
(6.73)\end{array}$ & 92.47 \\
\hline 2 & Fipronil $0.3 \mathrm{GR}(8 \mathrm{Kg} / \mathrm{ac})$ & $\begin{array}{c}19.12 \\
(25.90)\end{array}$ & $\begin{array}{c}6.22 \\
(14.29)\end{array}$ & $\begin{array}{c}3.17 \\
(10.15)\end{array}$ & $\begin{array}{c}4.11 \\
(11.65)\end{array}$ & 84.24 \\
\hline 3 & Cartap hydrochloride 4G ( $5 \mathrm{Kg} / \mathrm{ac}$ ) & $\begin{array}{c}19.80 \\
(26.35)\end{array}$ & $\begin{array}{c}6.47 \\
(14.66)\end{array}$ & $\begin{array}{c}4.00 \\
(11.49)\end{array}$ & $\begin{array}{c}3.99 \\
(11.46)\end{array}$ & 83.05 \\
\hline 4 & Chlorantraniliprole $18.5 \% \mathrm{SC}(60 \mathrm{ml} / \mathrm{ac})$ & $\begin{array}{c}18.83 \\
(25.68)\end{array}$ & $\begin{array}{c}9.54 \\
(17.95)\end{array}$ & $\begin{array}{c}7.91 \\
(16.29)\end{array}$ & $\begin{array}{c}7.04 \\
(15.35)\end{array}$ & 74.84 \\
\hline 5 & Fipronil 5SC (2 ml/lt) & $\begin{array}{c}20.74 \\
(27.03)\end{array}$ & $\begin{array}{c}9.65 \\
(17.89)\end{array}$ & $\begin{array}{c}8.07 \\
(16.43)\end{array}$ & $\begin{array}{c}7.40 \\
(15.74)\end{array}$ & 74.54 \\
\hline 6 & Chlorantraniliprole $18.5 \% \mathrm{SC}(150 \mathrm{ml} / \mathrm{ac})$ & $\begin{array}{c}20.41 \\
(26.79)\end{array}$ & $\begin{array}{c}4.02 \\
(11.53)\end{array}$ & $\begin{array}{c}1.09 \\
(5.97)\end{array}$ & $\begin{array}{c}1.26 \\
(6.42)\end{array}$ & 92.10 \\
\hline 7 & Flubendiamide $480 \mathrm{SC}(0.075 \mathrm{ml} / \mathrm{lt})$ & $\begin{array}{c}19.79 \\
(26.35)\end{array}$ & $\begin{array}{c}13.13 \\
(21.10)\end{array}$ & $\begin{array}{c}10.09 \\
(18.49)\end{array}$ & $\begin{array}{c}11.87 \\
(19.95)\end{array}$ & 67.47 \\
\hline 8 & Chlorpyriphos $20 \mathrm{EC}$ (2 ml/lt) & $\begin{array}{c}19.90 \\
(26.46)\end{array}$ & $\begin{array}{c}14.92 \\
(22.68)\end{array}$ & $\begin{array}{c}12.08 \\
(20.30)\end{array}$ & $\begin{array}{c}15.41 \\
(23.05)\end{array}$ & 67.07 \\
\hline 9 & Emamectin benzoate $0.5 \mathrm{SG}(0.2 \mathrm{~g} / \mathrm{lt})$ & $\begin{array}{c}20.21 \\
(26.65)\end{array}$ & $\begin{array}{c}13.20 \\
(21.21)\end{array}$ & $\begin{array}{c}9.70 \\
(18.10)\end{array}$ & $\begin{array}{c}12.16 \\
(20.35)\end{array}$ & 69.87 \\
\hline 10 & Untreated check & $\begin{array}{c}20.40 \\
(26.77)\end{array}$ & $\begin{array}{c}23.98 \\
(29.29)\end{array}$ & $\begin{array}{c}27.35 \\
(31.48)\end{array}$ & $\begin{array}{c}29.85 \\
(32.82)\end{array}$ & 61.88 \\
\hline & SEm \pm & 1.27 & 1.26 & 1.54 & 0.70 & 1.15 \\
\hline & CD (0.05) & 3.77 & 3.74 & 1.54 & 2.08 & 3.43 \\
\hline
\end{tabular}

Values are mean of three replications; Figures in the parenthesis are arc sin transferred value. 
Table.2 Cost economics of different molecules tested against Early Shoot borer on Sugarcane (ARS, Bidar)

\begin{tabular}{|c|c|c|c|c|c|c|c|c|c|}
\hline $\begin{array}{l}\text { Sl. } \\
\text { No. }\end{array}$ & Treaments & $\begin{array}{c}\text { Yield } \\
\text { t/ha }\end{array}$ & $\begin{array}{c}\text { Common } \\
\text { cost of } \\
\text { cultivation }\end{array}$ & $\begin{array}{c}\text { Treatment } \\
\text { cost }\end{array}$ & $\begin{array}{l}\text { Total } \\
\text { cost }\end{array}$ & $\begin{array}{l}\text { Gross } \\
\text { return }\end{array}$ & $\begin{array}{c}\text { Net } \\
\text { return }\end{array}$ & $\begin{array}{c}\text { Treatment } \\
\text { benefit } \\
\text { Rs. }\end{array}$ & $\begin{array}{l}\mathrm{B}: \mathrm{C} \\
\text { ratio }\end{array}$ \\
\hline 1 & $\begin{array}{l}\text { Chlorantraniliprole } 0.4 \text { GR (8 } \\
\mathrm{Kg} / \mathrm{ac})\end{array}$ & 92.47 & 43000 & 4200 & 47200 & 221928 & 174728 & 69216 & 3.70 \\
\hline 2 & Fipronil $0.3 \mathrm{GR}$ (8 Kg/ac) & 84.24 & 43000 & 2200 & 45200 & 202164 & 156964 & 51452 & 3.47 \\
\hline 3 & Cartap hydrochloride 4G ( $5 \mathrm{Kg} / \mathrm{ac}$ ) & 83.05 & 43000 & 1450 & 44450 & 199308 & 154858 & 49346 & 3.48 \\
\hline 4 & $\begin{array}{l}\text { Chlorantraniliprole } 18.5 \% \text { SC ( } 60 \\
\mathrm{ml} / \mathrm{ac})\end{array}$ & 74.84 & 43000 & 2300 & 45300 & 179616 & 134316 & 28804 & 2.97 \\
\hline 5 & Fipronil 5SC ( $2 \mathrm{ml} / \mathrm{lt})$ & 74.54 & 43000 & 1500 & 44500 & 178884 & 134384 & 28872 & 3.02 \\
\hline 6 & $\begin{array}{l}\text { Chlorantraniliprole } 18.5 \% \mathrm{SC}(150 \\
\mathrm{ml} / \mathrm{ac})\end{array}$ & 92.10 & 43000 & 4700 & 47700 & 221028 & 173328 & 67816 & 3.63 \\
\hline 7 & $\begin{array}{l}\text { Flubendiamide } 480 \mathrm{SC}(0.075 \\
\mathrm{ml} / \mathrm{lt})\end{array}$ & 67.47 & 43000 & 1850 & 44850 & 161916 & 117066 & 11554 & 2.61 \\
\hline 8 & Chlorpyriphos $20 \mathrm{EC} \mathrm{(2} \mathrm{ml/lt)}$ & 67.07 & 43000 & 1100 & 44100 & 160968 & 116868 & 11356 & 2.65 \\
\hline 9 & $\begin{array}{l}\text { Emamectin benzoate } 0.5 \mathrm{SG} \\
(0.2 \mathrm{~g} / \mathrm{lt})\end{array}$ & 69.87 & 43000 & 1900 & 44900 & 167688 & 122788 & 17276 & 2.73 \\
\hline 10 & Untreated check & 61.88 & 43000 & 0 & 43000 & 148512 & 105512 & 0 & 2.45 \\
\hline
\end{tabular}


Table.3 Evaluation of new molecules against Early Shoot borer on Sugarcane (2013-14 and 2014-15) at ARS, Kalburgi (Pooled)

\begin{tabular}{|c|c|c|c|c|c|c|}
\hline \multirow{2}{*}{$\begin{array}{l}\text { Sl. } \\
\text { No. }\end{array}$} & \multirow[t]{2}{*}{ Treatments } & \multicolumn{4}{|c|}{ Early shoot borer incidence (\% Dead heart) } & \multirow[t]{2}{*}{ Yield (t/ha) } \\
\hline & & 1DBS & 15 DAS & 30 DAS & 45 DAS & \\
\hline 1 & Chlorantraniliprole 0.4 GR ( $8 \mathrm{Kg} / \mathrm{ac})$ & $\begin{array}{c}23.90 \\
(29.27)\end{array}$ & $\begin{array}{c}5.05 \\
(12.99)\end{array}$ & $\begin{array}{c}1.24 \\
(6.38)\end{array}$ & $\begin{array}{c}1.56 \\
(7.17)\end{array}$ & $94.89 \mathrm{a}$ \\
\hline 2 & Fipronil 0.3 GR (8 Kg/ac) & $\begin{array}{c}24.00 \\
(29.33)\end{array}$ & $\begin{array}{c}7.53 \\
(15.92)\end{array}$ & $\begin{array}{c}3.85 \\
(11.32)\end{array}$ & $\begin{array}{c}4.95 \\
(12.86)\end{array}$ & $87.75 \mathrm{~b}$ \\
\hline 3 & Cartap hydrochloride 4G ( $5 \mathrm{Kg} / \mathrm{ac}$ ) & $\begin{array}{c}24.15 \\
(29.43)\end{array}$ & $\begin{array}{c}7.85 \\
(16.27)\end{array}$ & $\begin{array}{c}4.95 \\
(12.86)\end{array}$ & $\begin{array}{c}5.39 \\
(13.42)\end{array}$ & $86.95 \mathrm{~b}$ \\
\hline 4 & Chlorantraniliprole $18.5 \% \mathrm{SC}(60 \mathrm{ml} / \mathrm{ac})$ & $\begin{array}{c}22.78 \\
(28.51)\end{array}$ & $\begin{array}{c}11.23 \\
(19.58)\end{array}$ & $\begin{array}{c}9.68 \\
(18.12)\end{array}$ & $\begin{array}{c}8.70 \\
(17.15)\end{array}$ & $79.55 \mathrm{c}$ \\
\hline 5 & Fipronil 5SC (2 ml/lt) & $\begin{array}{c}23.90 \\
(29.27)\end{array}$ & $\begin{array}{c}10.91 \\
(19.29)\end{array}$ & $\begin{array}{c}8.86 \\
(17.31)\end{array}$ & $\begin{array}{c}7.80 \\
(16.22)\end{array}$ & $79.79 \mathrm{c}$ \\
\hline 6 & Chlorantraniliprole $18.5 \% \mathrm{SC}(150 \mathrm{ml} / \mathrm{ac})$ & $\begin{array}{c}23.40 \\
(28.93)\end{array}$ & $\begin{array}{c}5.33 \\
(13.34)\end{array}$ & $\begin{array}{c}1.28 \\
(6.48)\end{array}$ & $\begin{array}{c}1.38 \\
(6.73)\end{array}$ & $95.61 \mathrm{a}$ \\
\hline 7 & Flubendiamide $480 \mathrm{SC}(0.075 \mathrm{ml} / \mathrm{lt})$ & $\begin{array}{c}23.03 \\
(28.68)\end{array}$ & $\begin{array}{c}14.93 \\
(22.73)\end{array}$ & $\begin{array}{c}11.28 \\
(19.62)\end{array}$ & $\begin{array}{c}13.68 \\
(21.70)\end{array}$ & $76.23 \mathrm{~d}$ \\
\hline 8 & Chlorpyriphos $20 \mathrm{EC}(2 \mathrm{ml} / \mathrm{lt})$ & $\begin{array}{c}22.90 \\
(28.59)\end{array}$ & $\begin{array}{c}17.33 \\
(24.60)\end{array}$ & $\begin{array}{c}13.85 \\
(21.85)\end{array}$ & $\begin{array}{c}18.25 \\
(25.29)\end{array}$ & $74.84 \mathrm{~d}$ \\
\hline 9 & Emamectin benzoate $0.5 \mathrm{SG}(0.2 \mathrm{~g} / \mathrm{lt})$ & $\begin{array}{c}23.48 \\
(28.98)\end{array}$ & $\begin{array}{c}15.33 \\
(23.05)\end{array}$ & $\begin{array}{c}11.32 \\
(19.66)\end{array}$ & $\begin{array}{c}14.20 \\
(22.14)\end{array}$ & $75.08 \mathrm{~d}$ \\
\hline 10 & Untreated check & $\begin{array}{c}21.75 \\
(27.80)\end{array}$ & $\begin{array}{c}23.86 \\
(29.24)\end{array}$ & $\begin{array}{c}28.05 \\
(31.98)\end{array}$ & $\begin{array}{c}31.35 \\
(34.05)\end{array}$ & $69.01 \mathrm{e}$ \\
\hline & SEm \pm & 0.81 & 0.53 & 0.59 & 0.57 & 0.98 \\
\hline & CD (0.05) & NS & 1.58 & 1.78 & 1.70 & 3.01 \\
\hline
\end{tabular}

Values are mean of three replications; Figures in the parenthesis are arc sin transferred value. 
Table.4 Cost economics of different molecules tested against Early Shoot borer on Sugarcane (ARS, Kalburgi)

\begin{tabular}{|c|c|c|c|c|c|c|c|c|c|}
\hline $\begin{array}{l}\text { Sl. } \\
\text { No. }\end{array}$ & Treaments & $\begin{array}{l}\text { Yield } \\
\text { t/ha }\end{array}$ & $\begin{array}{c}\text { Common } \\
\text { cost of } \\
\text { cultivation }\end{array}$ & $\begin{array}{l}\text { Treatment } \\
\text { cost }\end{array}$ & $\begin{array}{c}\text { Total } \\
\text { cost }\end{array}$ & $\begin{array}{l}\text { Gross } \\
\text { return }\end{array}$ & $\begin{array}{l}\text { Net } \\
\text { return }\end{array}$ & $\begin{array}{c}\text { Treatment } \\
\text { benefit } \\
\text { Rs. }\end{array}$ & $\begin{array}{l}\text { B:C } \\
\text { ratio }\end{array}$ \\
\hline 1 & Chlorantraniliprole 0.4 GR $(8 \mathrm{Kg} / \mathrm{ac})$ & 94.89 & 43000 & 4200 & 47200 & 227724 & 180524 & 57912 & 3.82 \\
\hline 2 & Fipronil $0.3 \mathrm{GR}(8 \mathrm{Kg} / \mathrm{ac})$ & 87.75 & 43000 & 2200 & 45200 & 210600 & 165400 & 42788 & 3.66 \\
\hline 3 & Cartap hydrochloride 4G ( $5 \mathrm{Kg} / \mathrm{ac}$ ) & 86.95 & 43000 & 1450 & 44450 & 208668 & 164218 & 41606 & 3.69 \\
\hline 4 & Chlorantraniliprole $18.5 \% \mathrm{SC}(60 \mathrm{ml} / \mathrm{ac})$ & 79.55 & 43000 & 2300 & 45300 & 190920 & 145620 & 23008 & 3.21 \\
\hline 5 & Fipronil 5SC (2 ml/lt) & 79.79 & 43000 & 1500 & 44500 & 191484 & 146984 & 24372 & 3.30 \\
\hline 6 & $\begin{array}{l}\text { Chlorantraniliprole } 18.5 \% \text { SC ( } 150 \\
\text { ml/ac) }\end{array}$ & 95.61 & 43000 & 4700 & 47700 & 229464 & 181764 & 59152 & 3.81 \\
\hline 7 & Flubendiamide $480 \mathrm{SC}(0.075 \mathrm{ml} / \mathrm{lt})$ & 76.23 & 43000 & 1850 & 44850 & 182940 & 138090 & 15478 & 3.08 \\
\hline 8 & Chlorpyriphos $20 \mathrm{EC}(2 \mathrm{ml} / \mathrm{lt})$ & 74.84 & 43000 & 1100 & 44100 & 179616 & 135516 & 12904 & 3.07 \\
\hline 9 & Emamectin benzoate $0.5 \mathrm{SG}(0.2 \mathrm{~g} / \mathrm{lt})$ & 75.08 & 43000 & 1900 & 44900 & 180180 & 135280 & 12668 & 3.01 \\
\hline 10 & Untreated check & 69.01 & 43000 & 0 & 43000 & 165612 & 122612 & 0 & 2.85 \\
\hline
\end{tabular}


Among all the treatments, at 15 Days After Spraying (DAS) Chloranthriniliprole 0.4 GR @ $8 \mathrm{~kg} / \mathrm{ac}$ and Chloranthriniliprole 18.5 SC @ $150 \mathrm{ml} / \mathrm{ac}$ were found to be significantly superior over all other treatment and recorded 5.05 and 5.33 per cent dead hearts respectively. The treatment with Fipronil 0.3 GR @ 8kg/ac and Cartaphydrochloride 4G @ $5 \mathrm{~kg} / \mathrm{ac}$ was next in order of efficacy with 7.53 and 7.85 per cent dead hearts respectively with no significant difference among them. The next best treatments in order of their efficacy were Fipronil 5 SC @ 2ml/lt, Chloranthriniliprole 18.5 SC @60 ml/ac, Flubendiamide 480 SC @ 0.075 ml/lt, Emamectin benzoate 0.5 SG @0.2g/lt, and Chlorpyriphos 20 EC @ 2 ml/lt with 10.91, $11.23,14.93,15.33$ and 17.33 per cent dead hearts respectively. The dead hearts percentage was significantly low in all the insecticidal treatments as compared to untreated control when observations recorded at 15 DAS with 23.86 per cent. The data on per cent dead hearts recorded at 30 DAS followed the same trend as that of 15 DAS.

The early shoot borer incidence recorded at forty five days after treatment showed the lowest early shoot borer incidence in the plots treated with Chlorantraniliprole 18.5 SC @ $150 \mathrm{ml} / \mathrm{ac}$ and Chlorantraniliprole 0.4 GR @ $8 \mathrm{~kg} / \mathrm{ac}$ and were found consistently to be the most promising treatment by recording significantly lowest 1.38 and 1.56 per cent dead hearts respectively.

The treatment with Fipronil 0.3 GR @ 8kg/ac and Cartaphydrochloride 4G @ 5kg/ac are the next in the order of efficacy with 4.95 and 5.39 per cent dead hearts respectively with no significant difference among them. The next best treatments in the order of their efficacy were Fipronil 5 SC @ 2ml/lt, Chlorantraniliprole 18.5 SC @ $60 \mathrm{ml} / \mathrm{ac}$, Flubendiamide 480 SC @ 0.075 ml/lt, Emamectin benzoate 0.5 SG @ 0.2g/lt, and
Chlorpyriphos 20 EC @ 2 ml/lt with 7.80, 8.70, 13.68, 14.20 and 18.25 per cent dead hearts respectively. However, untreated control plot recorded the highest per cent dead heart of 31.35 per cent.

\section{Yield}

The significantly highest cane yield was recorded in Chloranthriniliprole 18.5 SC @ $150 \mathrm{ml} / \mathrm{ac}$ and Chloranthriniliprole 0.4 GR @ $8 \mathrm{~kg} / \mathrm{ac}$ with 95.61 tonns/ha and 94.89 tonns/ha respectively. The next best treatments were Fipronil 0.3 GR @ 8kg/ac and Cartaphydrochloride 4G @5kg/ac which recorded 87.75 and 86.95 tonns/ha cane yield respectively.

These two treatments were followed by Fipronil 5 SC @ 2ml/lt, Chloranthriniliprole 18.5 SC @60 ml/ac, Flubendiamide 480 SC @ 0.075 ml/lt Emamectin benzoate 0.5 SG @ 0.2g/lt, and Chlorpyriphos 20 EC @ 2 ml/lt with the cane yield of 79.79, 79.55, 76.23, 75.08 and 74.84 tonns/ha respectively (Table $3)$. However, untreated control plot recorded the lowest yield of 69.01 tonns/ha.

\section{B: C ratio}

Chloranthriniliprole 0.4 GR @ 8 kg/ac and Chloranthriniliprole $18.5 \mathrm{SC} @ 150 \mathrm{ml} / \mathrm{ac}$ recorded highest $\mathrm{B}: \mathrm{C}$ ratio of 3.82 and 3.81 respectively (Table 3). The next best treatments were and Cartaphydrochloride 4G @ 5kg/ac and Fipronil 0.3 GR @ 8kg/ac which recorded B: C ratio of 3.69 and 3.66 respectively. These two treatments were followed by Fipronil 5 SC @ 2ml/lt, Chloranthriniliprole 18.5 SC @ $60 \mathrm{ml} / \mathrm{ac}$, Flubendiamide $480 \quad$ SC @ $0.075 \quad \mathrm{ml} / \mathrm{lt}$ Chlorpyriphos 20 EC @ 2 ml/lt and Emamectin benzoate $0.5 \mathrm{SG} @ 0.2 \mathrm{~g} / \mathrm{lt}$, with $3.30,3.21,3.08,3.07$ and $3.01 \mathrm{~B}: \mathrm{C}$ ratio respectively. However, untreated control plot recorded the lowest $\mathrm{B}$ : $\mathrm{C}$ ratio of 2.85 . 
The results of the present study reveals that the incidence of Early Shoot Borer per cent was more during 2014-15 compared to 201314 and also it was also observed that yield levels were low during 2013-14 compared to 2014-15. Further, no Phytotoxicity symptoms were noticed on the crop after imposing the treatments.

Looking in to the results of two seasons and two location data, Chloranthriniliprole 18.5 SC @ $150 \mathrm{ml} / \mathrm{ac}$ can be very effectively used for managing the Early Shoot Borer in sugarcane as they have recorded lowest percent incidence of early shoot borer and highest cane yield. The present findings are in accordance with Bhawar et al., 2016 who reported that, Rynoxpyr 20 SC was very effective and significantly superior over all other treatments in reducing the dead hearts to minimum level of 3.13 per cent. Sheeba Jasmine et al., (2012) and Gaje Singh et al., (2009) also postulated that rynaxpyr was most effective treatment recording the lowest incidence of sugarcane early shoot borer (15.43\%). The present findings are in accordance with the results of Padmasri et al., 2014 as they have reported that, Chloranthriniliprole $18.5 \mathrm{SC} @ 0.36 \mathrm{ml} / 1$ of water recorded the lowest per cent (11.07) early shoot borer incidence. The present findings are also in agreement with the observations of Singh et al., (2009) reported Rynaxypyr 20 Sc @100 g.a.i/ha was found to be the best with minimum of 0.12 per cent infestation by early shoot borer and gave maximum yield of 77.13 t/ha. Jaipal et al., (2010) recorded similar observations and reported that the mean incidence of shoot borer was lowered by 60 per cent due to Rynaxypyr 20 SC treatment given as root drench and registered significantly increased cane yield over the untreated control as well as the standard recommended insecticides Carbofuran. Pandey, 2014 reported that Chloranthriniliprole $20 \mathrm{SC}$ was most effective as it exhibited least dead hearts of $2.85 \%$ with the decrease of $83.43 \%$ in the incidence over control.

Further Chloranthriniliprole 0.4 GR @ 8 $\mathrm{kg} / \mathrm{ac}$ also found effective and on par with the Chloranthriniliprole18.5 SC @ $150 \mathrm{ml} / \mathrm{ac}$ in managing the early shoot borer damage. These results are in line with Umashankar et al., 2018 reported that the treatment with Chlorantraniliprole 0.4G @ 0.09 g a.i. /ha recorded lowest cumulative incidence (2.79 $\%)$ and highest per cent reduction over the control (85.78\%). Bhawar et al., 2015 also reported that the treatment with Fertera $0.4 \mathrm{G}$ @ $30 \mathrm{~g}$ ai/ha was found effective by recording 3.64 per cent dead heart.

The next best treatments were Fipronil 0.3 GR @ 8kg/ac and Cartaphydrochloride 4G @ $5 \mathrm{~kg} / \mathrm{ac}$ which recorded lowest per cent dead hearts without any significant difference among them. These findings are in accordance with Bhawar et al., 2015, who found that Fipronil $0.3 \mathrm{G} @ 7.5 \mathrm{~g}$ a.i/ha recorded 5.25 per cent dead hearts and found on par with Cartaphydrochloride 4G @ 750 g a. i./ha recording 5.44 per cent dead hearts and. Bhawar et al., (2016) who also reported that Flubendiamide $39.35 \mathrm{SC}$ and Cartap hydrochloride $4 \mathrm{G}$ were most effective in reducing the ESB attack.

Among all the treatments, Chloranthriniliprole 0.4 GR @ 8 kg/ac or Chloranthriniliprole 18.5 SC @ 150 ml/ac can be very effectively used for managing the Early Shoot Borer in sugarcane.

\section{References}

Avasthy, P.N. and Tiwari, N.K. (1986). The shoot borer, Chilo infuscatellus snellen. In: "Sugarcane Entomology in India" (Eds. David, H.S., Easwaramoorthy and 
Jayanthi, R.). Sugarcane Breeding Institute, Coimbatore, pp. 69-92.

Bhawar, N. Mohite, P. and Patil, S. (2016). Bioefficacy of new insecticide molecules against Sugarcane Early Shoot borer Chilo infuscatellus (Snellen) in Kolhapur Region of Maharashtra. J. Agr. Vet. Sci., 9(1): 3235.

Gaje Singh, C. S. Prasad, Anil Sirohi, Arvind Kumar and Nawab. Ali, (2009). Field evaluation of Rynaxypr 20 SC against Insect Pest Sugarcane. Ann.pl. Protec. Sci. 17(1):50-52.

Gupta, S.C., Yazdani, S.S., Hameed, S.F. and Agarwal, M.L. (1993). Losses in sugarcane by different broods of top borer, Scirpophaga excerptalis Walker in northern Bihar. J. Insect Sci., 6(1): $125-127$.

Jaipal, S., Chaudary, O.P.S., and Prasad, R. (2010). Evaluation of rynaxypyr (Coregen) $20 \mathrm{SC}$ for the management of early shoot borer and top shoot borer in sugarcane. Indian journal of sugarcane technology, 25(1\&2):47-50.

Krishnanurthy Rao, B.H. (1954). Apparent and actual yield of sugar cane and the part played by stem borers. Proc.
A.Conv. Sug. Technol. Assoc. India, 23: 25-27.

Padmasri, A., Vidyasagar, G.E., and Bharathi, V. (2014). Evaluation of new molecules for management of stem borer on sugarcane. J. Agric. Vet. Sci., 7(6): 40 42.

Pandey, S. K. (2014). Comparative efficacy of some insecticides on early shoot borer (Chilo infuscatellus Snellen) incidence in sugarcane under subtropical India. Vegetos, 27 (1): 146148.

Sheeba J. R., Rajendran, B and kanchana Rani, (2012). Biological Integrated Pest Management of sugarcane. Journal of Entomology, 5: 209-211.

Singh, G., Prasad, C.S., Sirohi, A., Kumar, A. and Ali, N. (2009). Field evaluation of Rynaxypyr 20SC against insect pests of sugarcane. Annuals of plant protection sciences, 17(1):75-79.

Umashankar, H. G., Patel, V.N., Nagaraja, T., Vijaykumar, L. and Sugeetha, S. (2018). Evaluation of new insecticide molecules for their effectiveness in the management of sugarcane early shoots borer, Chilo infuscatellus (Snellen). Journal of Applied and Natural Science, 10 (1): $434-438$.

\section{How to cite this article:}

Shobharani, M., V. Rachappa, Sidramappa and Sunilkumar, N.M. 2018. Evaluation of New Insecticide Molecules for the Management of Early Shoot Borer (Chilo infuscatellus (Snellen) on Sugarcane. Int.J.Curr.Microbiol.App.Sci. 7(09): 2079-2088.

doi: https://doi.org/10.20546/ijcmas.2018.709.253 